\title{
INTRODUCTORY COMMENTS FOR THE 2018 EDITORS' CHOICE ISSUE
}

\author{
SUSAN FRIEDLANDER
}

This issue of the Bulletin of the American Mathematical Society is dedicated to the memory of Andrew Ranicki who was for many years a member of the Bulletin Editorial Board. He made very valuable contributions to a number of special issues, including the 2011 Editors' Choice issue. Andrew will be much missed by all who knew him.

The first issue of the Bulletin was published in 1891, which makes it not only the original journal of the AMS but also one of the older prestigious journals covering a broad range of mathematics in the world. Since its inception the Bulletin has published a treasure trove of influential seminal articles. I invited Bulletin editors, some of whom have joined the board in the past few years, to browse the Bulletin archives which are freely available to everyone on the web. Each editor selected an article which was particularly meaningful to him or her and wrote a short commentary about their selected article. This present issue contains a reprinting of seven such articles along with commentaries and, in two cases, extra illuminating material.

Mark Goresky chose André Weil's article "Numbers of solutions of equations in finite fields" which appeared in 1949. This remarkable article introduced the Weil conjectures to the world and initiated an amazingly rich area of mathematics. The "Selected Mathematical Reviews" in the Mathematical Perspectives section of this issue are devoted to results concerning some of the Weil conjectures and related developments.

Maciej Zworski chose Stephen Smale's article "Differentiable dynamical systems" which appeared in 1967. This article not only gave an extensive survey of global analysis defined by differentiable dynamical systems but also paved the way for exciting new directions. One example is Smale's conjecture about the meromorphic continuation of dynamical zeta functions of flows. This conjecture has very recently been resolved by Semyon Dyatlov and Colin Guillarmou. Their short proof is published as an afterword to Smale's original article.

Bryna Kra chose what became a very influential research announcement by Donald Ornstein and Benjamin Weiss on the ergodic theory of amenable group actions: the Rohlin lemma.

Irene Gamba chose Cathleen Morawetz's article based on her 1981 Gibbs Lecture entitled "The mathematical approach to the sonic barrier". This article combined state-of-the-art applied mathematics with practical issues concerning flight at close to the speed of sound. It is still a rich source of open questions.

Burt Totaro chose Barry Mazur's famous survey article entitled "Arithmetic on curves" which was published in 1986. It gives a grand survey of the central 
themes in number theory up to the 1980s. We are delighted that Barry Mazur has written a short afterword mentioning some major advances in the subject during the intervening three decades.

William Minicozzi chose Brian White's article "Nonunique tangent maps at isolated singularities of harmonic maps" which was published in 1992. This short article emphasized the subtlety of uniqueness in understanding singularities in geometry and analysis.

Ivan Corwin chose the article by David Aldous and Persi Diaconis entitled "Longest increasing subsequences: from patience sorting to the Baik-Deift-Johansson theorem" which was published in 1999. The past two decades have seen an explosion of developments in many areas of mathematics following the publication of this paper. One such hot topic is Kardar-Parisi-Zhang (KPZ) universality which predicts long time behavior of many disparate systems.

We hope that you will enjoy reading these personal selections of the editors. We are always seeking submissions of excellent survey articles that will become future classics in the spirit of the diverse and influential articles that are reprinted in this issue.

Department of Mathematics, University of Southern California, Los Angeles, CalIFORNIA 